\title{
INVESTIGATION OF HM-NETWORK WITH PRIORITY MESSAGES AND DEPENDING ON TIME INCOMES FROM TRANSITIONS BETWEEN ITS STATES
}

\author{
Olga Kiturko ${ }^{1}$, Mikhail Matalytski ${ }^{2}$ \\ ${ }^{1}$ Faculty of Mathematics and Computer Science, Grodno State University \\ Grodno, Belarus \\ ${ }^{2}$ Institute of Mathematics, Czestochowa University of Technology \\ Częstochowa, Poland \\ Isytaya_om@mail.ru, ${ }^{2}$ m.matalytski@gmail.com
}

\begin{abstract}
In the article the technique of finding the expected income in multipleline queueing systems closed exponential by HM-network with priority messages in a case when the income from transitions between its states is random variables with known average values is described. The intensity of the service of messages in systems linearly depends on their number. The approached expressions for the expected income, depending on the average numbers of messages in systems are received. For them the system of the linear differential equations with broken right parts is made. The example is calculated.
\end{abstract}

Keywords: HM-network, incomes, priority messages

\section{Introduction}

Markov HM queueing networks with priority messages in a case when the income from transitions between their states is the determined functions depending on states and time were considered in article [1]. Systems of the differencedifferential equations for the expected income of queueing systems (QS) of a network were received and were reduced to systems of the homogeneous ordinary differential equations which were solved with the help of differential schemes. In this article the expressions for the expected income of such HM network are received, but in a case when the income from transitions between its states is random variables $(\mathrm{RV})$ with a given expectation.

Let's consider the closed exponential HM (Howard-Matalytski) - network with the priority messages, consisting of $n$ QS $S_{1}, S_{2}, \ldots, S_{n}$. As a state of a network we will understand a vector $k(t)=(k, t)=\left(k_{11}(t), k_{12}(t) ; k_{21}(t), k_{22}(t) ; \ldots ; k_{n 1}(t), k_{n 2}(t)\right)$, where $k_{i 1}(t), k_{i 2}(t)$ - accordingly the number of priority (the first type) and usual (the second type) messages in system $S_{i}$ in an instant $t, i=\overline{1, n}$. Priority messages have an absolute priority in relation to usual messages [1,2]. Let $K_{1}(t)$ and $K_{2}(t)$ - accordingly the number of priority and usual messages served in a network, 
$K(t)=K_{1}(t)+K_{2}(t)$ - the total number of messages in a network; $m_{i}$ - the number of service lines of messages of type $s$ in QS $S_{i} ; p_{i j}$ - probability of messages transition after service from QS $S_{i}$ in system $S_{j} ; \mu_{i s}\left(k_{i s}(t)\right)$ - intensity of messages service of type $s$ in QS $S_{i}, i, j=\overline{1, n}, s=1,2$.

Let's assume that at any moment $t$ are satisfied conditions:

$$
k_{i 1}(t)<m_{i}, i=\overline{1, n} .
$$

Let's also enter the following designations:

$$
\begin{aligned}
& \varepsilon_{i 1}\left(k_{i 1}(t)\right)=\min \left\{k_{i 1}(t), m_{i}\right\}=k_{i 1}(t), i=\overline{1, n}, \\
& \varepsilon_{i 2}\left(k_{i 1}(t), k_{i 2}(t)\right)=\left\{\begin{array}{l}
k_{i 2}(t), k_{i 1}(t)+k_{i 2}(t)<m_{i}, \\
m_{i}-k_{i 1}(t), \quad k_{i 1}(t)+k_{i 2}(t) \geq m_{i}
\end{array}=\min \left\{k_{i 2}(t), m_{i}-k_{i 1}(t)\right\}, i=\overline{1, n} .\right.
\end{aligned}
$$

Let's find expressions for the expected income of network systems $S_{i}, i=\overline{1, n}$.

\section{Expected incomes of network systems}

Let's consider the dynamics of change of the income of some system $S_{i}$ of a network. Let's designate through $V_{i}(t)$ its income in an instant $t$. Let $V_{i}(0)=v_{i 0}$. The income of this QS in a instant $t+\Delta t$ can be submitted in a look $V_{i}(t+\Delta t)=$ $=V_{i}(t)+\Delta V_{i}(t, \Delta t)$. For finding $\Delta V_{i}(t, \Delta t)$ we will write out conditional probabilities of events which can occur during the time $\Delta t$ and changes of the income of systems $S_{i}$, connected with these events.

1) With probability $\mu_{j 1}\left(k_{j 1}(t)\right) k_{j 1}(t) u\left(k_{j 1}(t)\right) p_{j i} \Delta t+o(\Delta t)$ the message of the first type will pass after service from system $S_{j}$ to system $S_{i}$, thus the income of system $S_{i}$ will increase at a size $r_{j i}{ }^{(1)}$, and the income of system $S_{j}$ will decrease by this size, $r_{j i}{ }^{(1)}$ where $r_{j i}{ }^{(1)} \mathrm{RV}$ with expectation (exp.) $d_{j i}$.

2) With probability $\mu_{i 1}\left(k_{i 1}(t)\right) k_{i 1}(t) u\left(k_{i 1}(t)\right) p_{i j} \Delta t+o(\Delta t)$ the message of the first type will pass after service from system $S_{i}$ to system $S_{j}$, thus the income of system $S_{i}$ will decrease by size $R_{i j}{ }^{(1)}$, and the income of system $S_{j}$ will increase by this size, $i, j=\overline{1, n}, j \neq i$, where $R_{i j}^{\left({ }^{(1)}\right.} \mathrm{RV}$ with exp. $d_{i j}$.

3) With probability $\mu_{j 2}\left(k_{j 2}(t)\right) u\left(k_{j 2}(t)\right) \min \left\{k_{j 2}(t), m_{j}-k_{j 1}(t)\right\} p_{j i} \Delta t+o(\Delta t)$ the message of the second type will pass after service from system $S_{j}$ to system $S_{i}$, thus the income of system $S_{i}$ will increase at a size $r_{j i}{ }^{(2)}$, and the income of system $S_{j}$ will decrease by this size, $i, j=\overline{1, n}, j \neq i$, where $r_{j i}{ }^{(2)} \mathrm{RV}$ with exp. $b_{j i}$. 
4) With probability $\mu_{i 2}\left(k_{i 2}(t)\right) u\left(k_{i 2}(t)\right) \min \left\{k_{i 2}(t), m_{i}-k_{i 1}(t)\right\} p_{i j} \Delta t+o(\Delta t) \quad$ the message of the second type will pass after service from system $S_{i}$ to system $S_{j}$, thus the income of system $S_{i}$ will decrease by size $R_{i j}{ }^{(2)}$, and the income of system $S_{j}$ will increase by this size, $i, j=\overline{1, n}, j \neq i$, where $R_{i j}{ }^{(2)} \mathrm{RV}$ with exp. $b_{i j}$.

5) On an interval of time size $\Delta t$ the change of a state of system $S_{i}$ won't happen to $\quad 1-\sum_{\substack{j=1, j \neq i}}^{n}\left[\mu_{j 1}\left(k_{j 1}(t)\right) k_{j 1}(t)+\min \left\{k_{j 2}(t), m_{j}-k_{j 1}(t)\right\} \times\right.$
$\left.\times \mu_{j 2}\left(k_{j 2}(t)\right)\right] p_{j i} \Delta t-\sum_{\substack{j=1, j \neq i}}^{n}\left[\mu_{i 1}\left(k_{i 1}(t)\right) k_{i 1}(t)+\mu_{i 2}\left(k_{i 2}(t)\right) \min \left\{k_{i 2}(t), m_{i}-k_{i 1}(t)\right\}\right] p_{i j} \Delta t+$ $+o(\Delta t)$. Besides, for each small period $\Delta t$ the system $S_{i}$ increases the income by size $r_{i} \Delta t$, where $r_{i}$ - RV with exp. $c_{i}, i=\overline{1, n}$.

Let's also consider that the above-mentioned RV are in pairs independent, it is obvious that $r_{j i}^{(1)}=R_{i j}^{(1)}, r_{j i}^{(2)}=R_{i j}^{(2)}$ with probability 1 , i.e. $d_{j i}=d_{i j}, b_{j i}=b_{i j}, i, j=\overline{1, n}$. From the above follows:

$$
\Delta V_{i}(t, \Delta t)=
$$

$$
=\left\{\begin{array}{cl}
r_{j i}{ }^{(1)} \Delta t+r_{i} \Delta t & \text { with probability } \mu_{j 1}\left(k_{j 1}(t)\right) u\left(k_{j 1}(t)\right) k_{j 1}(t) p_{j i} \Delta t+o(\Delta t), \\
r_{j i}{ }^{(2)} \Delta t+r_{i} \Delta t & \text { with probability } \mu_{j 2}\left(k_{j 2}(t)\right) u\left(k_{j 2}(t)\right) \min \left\{k_{j 2}(t), m_{j}-k_{j 1}(t)\right\} p_{j i} \Delta t+o(\Delta t), \\
-R_{i j}{ }^{(1)} \Delta t+r_{i} \Delta t & \text { with probability } \mu_{i 1}\left(k_{i 1}(t)\right) u\left(k_{i 1}(t)\right) k_{i 1}(t) p_{i j} \Delta t+o(\Delta t), \\
-R_{i j}{ }^{(2)} \Delta t+r_{i} \Delta t & \text { with probability } \mu_{i 2}\left(k_{i 2}(t)\right) u\left(k_{i 2}(t)\right) \min \left\{k_{i 2}(t), m_{i}-k_{i 1}(t)\right\} p_{i j} \Delta t+o(\Delta t), \\
& \text { with probability } 1-\sum_{\substack{j=1, j \neq i}}^{n}\left[\mu_{j 1}\left(k_{j 1}(t)\right) k_{j 1}(t)+\mu_{j 2}\left(k_{j 2}(t)\right) \times\right. \\
r_{i} \Delta t & \left.\times \min \left\{k_{j 2}(t), m_{j}-k_{j 1}(t)\right\}\right] p_{j i} \Delta t-\sum_{\substack{j=1, j \neq i}}^{n}\left[\mu_{i 1}\left(k_{i 1}(t)\right) k_{i 1}(t)+\right. \\
& \left.+\mu_{i 2}\left(k_{i 2}(t)\right) \min \left\{k_{i 2}(t), m_{i}-k_{i 1}(t)\right\}\right] p_{i j} \Delta t+o(\Delta t) .
\end{array}\right.
$$

At the fixed realization of process $k(t)$ it is possible to write down:

$$
\begin{gathered}
M\left\{\Delta V_{i}(t, \Delta t) / k(t)\right\}=\left(\sum_{\substack{j=1 \\
j \neq i}}^{n} \mu_{j 1}\left(k_{j 1}(t)\right) u\left(k_{j 1}(t)\right) k_{j 1}(t) p_{j i} d_{j i}-\right. \\
-\sum_{\substack{j=1 \\
j \neq i}}^{n} \mu_{i 1}\left(k_{i 1}(t)\right) u\left(k_{i 1}(t)\right) k_{i 1}(t) p_{i j} d_{i j}+
\end{gathered}
$$




$$
\begin{gathered}
+\sum_{\substack{j=1 \\
j \neq i}}^{n} \mu_{j 2}\left(k_{j 2}(t)\right) u\left(k_{j 2}(t)\right) \min \left\{k_{j 2}(t), m_{j}-k_{j 1}(t)\right\} p_{j i} b_{j i}- \\
\left.-\sum_{\substack{j=1 \\
j \neq i}}^{n} \mu_{i 2}\left(k_{i 2}(t)\right) u\left(k_{i 2}(t)\right) \min \left\{k_{i 2}(t), m_{i}-k_{i 1}(t)\right\} p_{i j} b_{i j}+c_{i}\right) \Delta t+o(\Delta t) .
\end{gathered}
$$

Averaging $k(t)$ taking into account a normalization state $\sum_{k} P(k(t)=k)=1$, for the change of the expected income of system $S_{i}$ we receive

$$
\begin{gathered}
M\left\{\Delta V_{i}(t, \Delta t)\right\}=\sum_{k} P(k(t)=k) M\left\{\Delta V_{i l}(t, \Delta t) / k(t)\right\}= \\
=\sum_{k} P(k(t)=k)\left[\sum_{\substack{j=1 \\
j \neq i}}^{n} \mu_{j 1}\left(k_{j 1}(t)\right) u\left(k_{j 1}(t)\right) k_{j 1}(t) p_{j i} d_{j i}-\sum_{\substack{j=1 \\
j \neq i}}^{n} \mu_{i 1}\left(k_{i 1}(t)\right) u\left(k_{i 1}(t)\right) k_{i 1}(t) p_{i j} d_{i j}+\right. \\
-\sum_{\substack{j=1 \\
j \neq i}}^{n} \mu_{i 2}\left(k_{i 2}(t)\right) u\left(k_{i 2}(t)\right) \min \left\{k_{i 2}(t), m_{i}-k_{i 1}(t)\right\} p_{i j} b_{i j}+ \\
\left.+\sum_{\substack{j=1 \\
j \neq i}}^{n} \mu_{j 2}\left(k_{j 2}(t)\right) u\left(k_{j 2}(t)\right) \min \left\{k_{j 2}(t), m_{j}-k_{j 1}(t)\right\} p_{j i} b_{j i}+c_{i}\right] \Delta t+o(\Delta t) .
\end{gathered}
$$

It is obvious that it is possible to remove Heaviside's functions $u\left(k_{j 1}(t)\right)$, $u\left(k_{i 2}(t)\right)$ owing to their definition and existence of other corresponding factors in this expression.

Let's enter designation $v_{i}(t)=M\left\{V_{i}(t)\right\}, i=\overline{1, n}$. Let's have then

$$
\begin{gathered}
v_{i}(t+\Delta t)=v_{i}(t)+M\left\{\Delta V_{i}(t, \Delta t)\right\}= \\
=v_{i}(t)+\sum_{k} P(k(t)=k)\left[\sum_{\substack{j=1 \\
j \neq i}}^{n} \mu_{j 1}\left(k_{j 1}(t)\right) k_{j 1}(t) p_{j i} d_{j i}-\sum_{\substack{j=1 \\
j \neq i}}^{n} \mu_{i 1}\left(k_{i 1}(t)\right) k_{i 1}(t) p_{i j} d_{i j}-\right. \\
-\sum_{\substack{j=1 \\
j \neq i}}^{n} \mu_{i 2}\left(k_{i 2}(t)\right) \min \left\{k_{i 2}(t), m_{i}-k_{i 1}(t)\right\} p_{i j} b_{i j}+ \\
\left.+\sum_{\substack{j=1 \\
j \neq i}}^{n} \mu_{j 2}\left(k_{j 2}(t)\right) \min \left\{k_{j 2}(t), m_{j}-k_{j 1}(t)\right\} p_{j i} b_{j i}+c_{i}\right] \Delta t+o(\Delta t) .
\end{gathered}
$$

Further, passing to a limit at $\Delta t \rightarrow 0$, we will receive inhomogeneous linear ordinary differential equations (ODE) of the first order. 


$$
\begin{aligned}
\frac{d v_{i}(t)}{d t}= & \sum_{k} P(k(t)=k)\left[\sum_{\substack{j=1 \\
j \neq i}}^{n} \mu_{j 1}\left(k_{j 1}(t)\right) k_{j 1}(t) p_{j i} d_{j i}-\sum_{\substack{j=1 \\
j \neq i}}^{n} \mu_{i 1}\left(k_{i 1}(t)\right) k_{i 1}(t) p_{i j} d_{i j}-\right. \\
& -\sum_{\substack{j=1 \\
j \neq i}}^{n} \mu_{i 2}\left(k_{i 2}(t)\right) \min \left\{k_{i 2}(t), m_{i}-k_{i 1}(t)\right\} p_{i j} b_{i j}+ \\
& \left.+\sum_{\substack{j=1 \\
j \neq i}}^{n} \mu_{j 2}\left(k_{j 2}(t)\right) \min \left\{k_{j 2}(t), m_{j}-k_{j 1}(t)\right\} p_{j i} b_{j i}+c_{i}\right], i=\overline{1, n} .
\end{aligned}
$$

Having set initial states $v_{i}(0)=v_{i 0}, i=\overline{1, n}$, it is possible to find the expected income of systems of a network.

Let's consider that the intensity of a service of messages $\mu_{i s}\left(k_{i s}\right)$ linearly depend on the number of messages of type $s$ in system $S_{i}, i=\overline{1, n}, s=1,2$. In this case

$$
\begin{gathered}
\mu_{i 1}\left(k_{i 1}(t)\right)=\left\{\begin{array}{ll}
\mu_{i 1} k_{i 1}(t), & k_{i 1}(t) \leq m_{i}, \\
\mu_{i 1} m_{i}, & k_{i 1}(t)>m_{i},
\end{array}=\mu_{i 1} \min \left(k_{i 1}(t), m_{i}\right), i=\overline{1, n},\right. \\
\mu_{i 2}\left(k_{i 2}(t)\right)= \begin{cases}\mu_{i 2} k_{i 2}(t), k_{i 1}(t)+k_{i 2}(t)<m_{i}, \\
\mu_{i 2}\left(m_{i}-k_{i 1}(t)\right), k_{i 1}(t)+k_{i 2}(t) \geq m_{i}, \\
0, & k_{i 1}(t) \geq m_{i},\end{cases}
\end{gathered}
$$

i.e.

$$
\mu_{i 2}\left(k_{i 2}(t)\right)=\left\{\begin{array}{l}
\mu_{i 2} \min \left(k_{i 2}(t), m_{i}-k_{i 1}(t)\right\}, k_{i 1}(t)<m_{i}, \quad i=\overline{1, n} \\
0, k_{i 1}(t) \geq m_{i},
\end{array}\right.
$$

In view of states (1), we receive

$$
\mu_{i 1}\left(k_{i 1}(t)\right)=\mu_{i 1} k_{i 1}(t), \mu_{i 2}\left(k_{i 2}(t)\right)=\mu_{i 2} \min \left\{k_{i 2}(t), m_{i}-k_{i 1}(t)\right\}
$$

Function $y=\min \left(x_{i}, m_{i}\right)$ is convex up and therefore from Iyensen's inequality follows that $M \min \left(k_{i s}(t), m_{i}\right) \leq \min \left(N_{i s}(t), m_{i}\right)$, and this equality is reached when $N_{i s}(t)=M\left\{k_{i s}(t)\right\}=k_{i s}(t), i=\overline{1, n}, s=1,2$. This state is satisfied, for example, when the flows of messages entering into a network are regular, and holding times of messages in systems are constants. Let's also note that this condition is satisfied when all QS of a network function are in conditions of small or high loading.

As approximation of average value of expressions $\mu_{i 1}\left(k_{i 1}(t)\right)$ and $\mu_{i 2}\left(k_{i 2}(t)\right)$ we will take $\mu_{i 1} N_{i 1}(t)$ and $\mu_{i 2} \min \left(N_{i 2}(t), m_{i}-N_{i 1}(t)\right)$, i.e. we believe that 


$$
M \min \left\{k_{i 2}(t), m_{i}-k_{i 1}(t)\right\}=\min \left(N_{i 2}(t), m_{i}-N_{i 1}(t)\right),
$$

where $N_{i 1}(t)$ and $N_{i 1}(t)$ - average of the messages, the first and second types respectively, (expecting and being served) in system $S_{i}$ in an instant $t, i=\overline{1, n}$. Taking into account this assumption we receive the following equations

$$
\begin{aligned}
\frac{d v_{i}(t)}{d t} & =\left[\sum_{\substack{j=1 \\
j \neq i}}^{n} \mu_{j 1} N_{j 1}^{2}(t) p_{j i} d_{j i}-\sum_{\substack{j=1 \\
j \neq i}}^{n} \mu_{i 1} N_{i 1}^{2}(t) p_{i j} d_{i j}-\right. \\
& -\sum_{\substack{j=1 \\
j \neq i}}^{n} \mu_{i 2} \min \left(N_{i 2}(t), m_{i}-N_{i 1}(t)\right) p_{i j} b_{i j}+ \\
+ & \left.\sum_{\substack{j=1 \\
j \neq i}}^{n} \mu_{j 2} \min \left(N_{j 2}(t), m_{j}-N_{j 1}(t)\right) p_{j i} b_{j i}+c_{i}\right], i=\overline{1, n},
\end{aligned}
$$

from where follows that

$$
v_{i}(t)=v_{i}(0)+\int_{0}^{t} f_{i}\left(N_{j 1}(x), N_{j 2}(x), j=\overline{1, n}\right) d x, i=\overline{1, n},
$$

where $f_{i}\left(N_{j 1}(x), N_{j 2}(x), j=\overline{1, n}\right)$ - the expression standing in the right part (2) at $t=x$.

\section{About finding the average number of messages in systems}

Let's designate through $\bar{\rho}_{i 1}(t)$ and $\bar{\rho}_{i 2}(t)$ - an average number of busy service lines by messages of the first type and the second type respectively in system $S_{i}$ in a instant $t, i=\overline{1, n}$. Then $\mu_{i 1} \bar{\rho}_{i 1}(t) \Delta t$ and $\mu_{i 2} \bar{\rho}_{i 2}(t) \Delta t$ - the average number of messages of the first and second types which have left system $S_{i}$ in time $\Delta t$, and $\sum_{\substack{j=1 \\ j \neq i}}^{n} \mu_{j 1} \bar{\rho}_{j 1}(t) p_{j i} \Delta t$ and $\sum_{\substack{j=1 \\ j \neq i}}^{n} \mu_{j 2} \bar{\rho}_{j 2}(t) p_{j i} \Delta t$ - average number of messages of the first and second types respectively, arrived in $S_{i}$ from other QS in time $\Delta t$. Therefore

$$
\begin{gathered}
N_{i 1}(t+\Delta t)-N_{i 1}(t)=\sum_{\substack{j=1 \\
j \neq i}}^{n} \mu_{j 1} \bar{\rho}_{j 1}(t) p_{j i} \Delta t-\mu_{i 1} \bar{\rho}_{i 1}(t) \Delta t, \\
N_{i 2}(t+\Delta t)-N_{i 2}(t)=\sum_{\substack{j=1 \\
j \neq i}}^{n} \mu_{j 2} \bar{\rho}_{j 2}(t) p_{j i} \Delta t-\mu_{i 2} \bar{\rho}_{i 2}(t) \Delta t, i=\overline{1, n},
\end{gathered}
$$


from $\Delta t \rightarrow 0$ where at systems the ODE follow for $N_{i 1}(t)$ and $N_{i 2}(t)$ :

$$
\begin{gathered}
\frac{d N_{i 1}(t)}{d t}=\sum_{\substack{j=1 \\
j \neq i}}^{n} \mu_{j 1} \bar{\rho}_{j 1}(t) p_{j i}-\mu_{i 1} \bar{\rho}_{i 1}(t), \\
\frac{d N_{i 2}(t)}{d t}=\sum_{\substack{j=1 \\
j \neq i}}^{n} \mu_{j 2} \bar{\rho}_{j 2}(t) p_{j i}-\mu_{i 2} \bar{\rho}_{i 2}(t), i=\overline{1, n} .
\end{gathered}
$$

Sizes $\bar{\rho}_{i 1}(t)$ and $\bar{\rho}_{i 2}(t)$ can't precisely be found and therefore we approximate them in expressions

$$
\begin{aligned}
& \bar{\rho}_{i 1}(t)=\left\{\begin{array}{l}
N_{i 1}(t), N_{i 1}(t) \leq m_{i}, \\
m_{i}, N_{i 1}(t)>m_{i},
\end{array}=\min \left(N_{i 1}(t), m_{i}\right),\right. \\
& \bar{\rho}_{i 2}(t)=\left\{\begin{array}{l}
N_{i 2}(t), N_{i 2}(t) \leq m_{i}, \\
m_{i}, N_{i 2}(t)>m_{i},
\end{array}=\min \left(N_{i 2}(t), m_{i}\right) .\right.
\end{aligned}
$$

Then systems of the equations (4) and (5) will become

$$
\begin{gathered}
\frac{d N_{i 1}(t)}{d t}=\sum_{\substack{j=1 \\
j \neq i}}^{n} \mu_{j 1} \min \left(N_{j 1}(t), m_{i}\right) p_{j i}-\mu_{i 1} \min \left(N_{i 1}(t), m_{i}\right), i=\overline{1, n} . \\
\frac{d N_{i 2}(t)}{d t}=\sum_{\substack{j=1 \\
j \neq i}}^{n} \mu_{j 2} \min \left(N_{j 2}(t), m_{i}\right) p_{j i}-\mu_{i 2} \min \left(N_{i 2}(t), m_{i}\right), i=\overline{1, n} .
\end{gathered}
$$

These are systems linear the ODE with broken right parts. It is necessary to solve them by splitting of phase space into a number of areas and finding the decision in each of them. Systems (6) and (7) can be solved, for example, using a package of the applied Mathematica programs.

If the network functions so $\min \left(N_{i 1}(t), m_{i}\right)=N_{i 1}(t), \min \left(N_{i 2}(t), m_{i}\right)=N_{i 2}(t)$, $i=\overline{1, n}$, look like:

$$
\begin{gathered}
\frac{d N_{i 1}(t)}{d t}=\sum_{\substack{j=1 \\
j \neq i}}^{n} \mu_{j 1} N_{j 1}(t) p_{j i}-\mu_{i 1} N_{i 1}(t), \\
\frac{d N_{i 2}(t)}{d t}=\sum_{\substack{j=1 \\
j \neq i}}^{n} \mu_{j 2} N_{j 2}(t) p_{j i}-\mu_{i 2} N_{i 2}(t), i=\overline{1, n} .
\end{gathered}
$$


The last systems of the equations can be presented as a matrix

$$
\frac{d N_{s}(t)}{d t}=X_{s} N_{s}(t)
$$

where $N_{s}^{T}(t)=\left(N_{1 s}(t), N_{2 s}(t), \ldots, N_{n s}(t)\right), X_{s}$ - the square matrix consisting of elements $x_{s_{i j}}=\mu_{j s} p_{j i}$, if to put $p_{i i}=-1, i, j=\overline{1, n}, s=1,2$. The decision of system (10) looks like

$$
N_{s}(t)=N_{s}(0) e^{X_{s} t},
$$

where $N_{s}(0)$ - some set initial states, however, finding of elements of a matrix $e^{X_{s} t}$ is a complex task even for rather small values $n$.

\section{Example}

Consider the network shown in Figure 1. Let $\mu_{11}=1, \mu_{21}=2, \mu_{31}=3, \mu_{41}=4$, $\mu_{51}=5, \mu_{61}=6, \mu_{71}=7, \mu_{81}=8, \mu_{91}=9, \mu_{10,1}=10, \mu_{11,1}=11, \mu_{12,1}=12, \mu_{13,1}=13$, $\mu_{14,1}=14, \quad \mu_{15,1}=15, \quad \mu_{16,1}=16, \quad \mu_{17,1}=17, \quad \mu_{12}=10, \quad \mu_{22}=14, \quad \mu_{32}=6, \quad \mu_{42}=8$, $\mu_{52}=10, \quad \mu_{62}=12, \quad \mu_{72}=14, \quad \mu_{82}=16, \quad \mu_{92}=8, \quad \mu_{10,2}=10, \quad \mu_{11,2}=12, \quad \mu_{12,2}=14$, $\mu_{13,2}=9, \quad \mu_{14,2}=8, \quad \mu_{15,2}=10, \quad \mu_{16,2}=12, \quad \mu_{17,2}=14$. Let also: $v_{10}=9, v_{20}=25$, $v_{30}=18, v_{40}=17, v_{50}=44, v_{60}=7, v_{70}=38, v_{80}=43, v_{90}=40, v_{10,0}=9, v_{11,0}=45$, $v_{12,0}=46, v_{13,0}=3, v_{14,0}=1, v_{15,0}=8, v_{16,0}=39, v_{17,0}=17 ; c_{1}=c_{7}=c_{10}=c_{13}=c_{14}=2$, $c_{2}=3, c_{3}=c_{9}=c_{11}=c_{12}=c_{15}=4, c_{4}=c_{8}=c_{16}=1, c_{5}=5, c_{6}=c_{17}=7$.

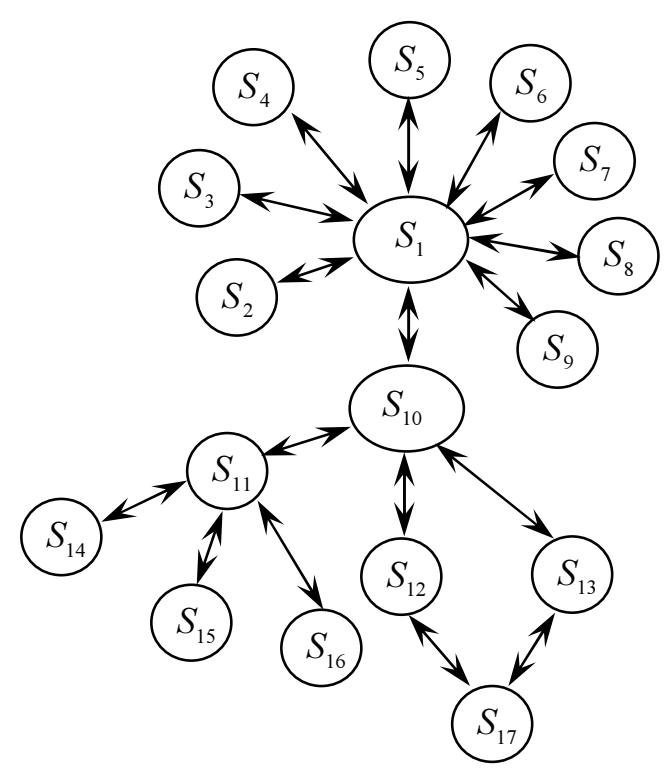

Fig. 1. Model of logistic transport system 
Expectations $d_{j i}$ and $b_{j i}$ we will reduce in matrices:

$$
D=\left\|d_{i j}\right\|_{17 \times 17}=\left(\begin{array}{ccccccccccccccccc}
2 & 3 & 1 & 1 & 1 & 4 & 4 & 4 & 3 & 5 & 5 & 4 & 6 & 4 & 2 & 1 & 7 \\
6 & 7 & 2 & 6 & 2 & 3 & 2 & 8 & 6 & 1 & 5 & 5 & 1 & 4 & 2 & 4 & 4 \\
2 & 7 & 9 & 5 & 5 & 8 & 8 & 7 & 7 & 8 & 1 & 5 & 3 & 4 & 1 & 6 & 1 \\
6 & 4 & 4 & 1 & 3 & 2 & 1 & 5 & 1 & 5 & 8 & 5 & 3 & 6 & 4 & 6 & 1 \\
4 & 5 & 7 & 3 & 8 & 3 & 9 & 6 & 6 & 5 & 2 & 1 & 3 & 3 & 3 & 2 & 8 \\
7 & 9 & 5 & 4 & 6 & 9 & 5 & 3 & 6 & 1 & 7 & 5 & 3 & 3 & 3 & 5 & 5 \\
5 & 6 & 5 & 2 & 2 & 5 & 9 & 7 & 9 & 9 & 3 & 9 & 6 & 8 & 9 & 8 & 3 \\
4 & 3 & 4 & 3 & 5 & 4 & 7 & 5 & 2 & 3 & 6 & 6 & 2 & 6 & 2 & 4 & 9 \\
5 & 6 & 8 & 7 & 5 & 7 & 9 & 2 & 8 & 3 & 9 & 9 & 9 & 3 & 3 & 9 & 1 \\
9 & 8 & 1 & 6 & 3 & 6 & 7 & 1 & 2 & 9 & 2 & 8 & 7 & 5 & 2 & 1 & 9 \\
2 & 7 & 6 & 4 & 3 & 7 & 2 & 5 & 6 & 6 & 1 & 5 & 1 & 5 & 3 & 7 & 7 \\
9 & 7 & 2 & 2 & 1 & 4 & 7 & 9 & 5 & 1 & 1 & 7 & 6 & 6 & 3 & 7 & 7 \\
1 & 9 & 4 & 9 & 9 & 8 & 5 & 9 & 2 & 8 & 7 & 6 & 2 & 8 & 8 & 2 & 2 \\
8 & 2 & 7 & 3 & 8 & 7 & 7 & 7 & 1 & 4 & 7 & 8 & 8 & 9 & 2 & 1 & 1 \\
3 & 2 & 2 & 8 & 7 & 6 & 4 & 1 & 1 & 8 & 4 & 1 & 8 & 4 & 6 & 1 & 8 \\
3 & 3 & 9 & 4 & 8 & 6 & 4 & 8 & 6 & 3 & 1 & 4 & 9 & 6 & 3 & 6 & 9 \\
4 & 4 & 6 & 3 & 7 & 3 & 2 & 5 & 5 & 9 & 1 & 3 & 8 & 9 & 3 & 7 & 6
\end{array}\right),
$$


Solving systems of (8) and (9) of ordinary differential equations of the first order with specified initial values used by the Runge-Kutta fourth-order, sold in the Mathematica 5.1 package. Then we substitute the solutions obtained in (3) we find the expected incomes network systems.

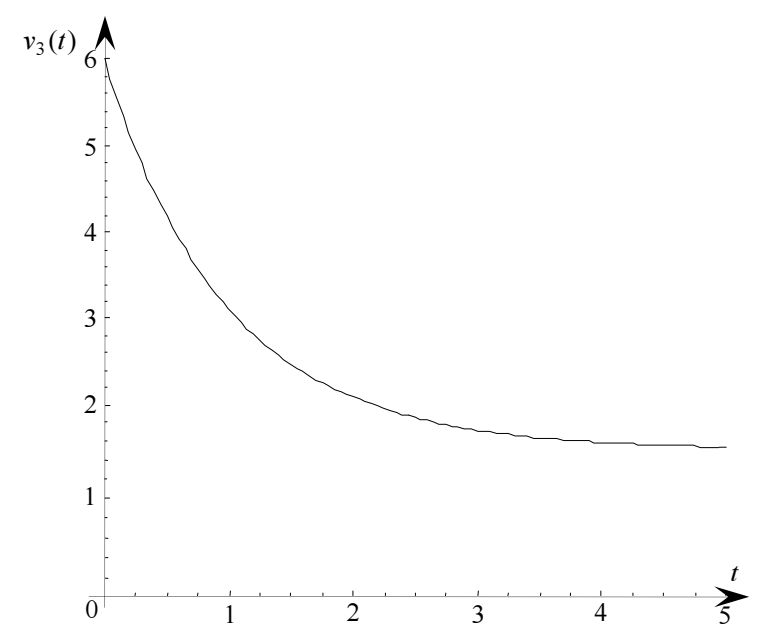

Fig. 2. Average of messages of system $S_{3}$ of the first type

For example, for system average number of messages of the first type it is presented in Figure 2, and the expected incomes for the system is represented in Figure 3.

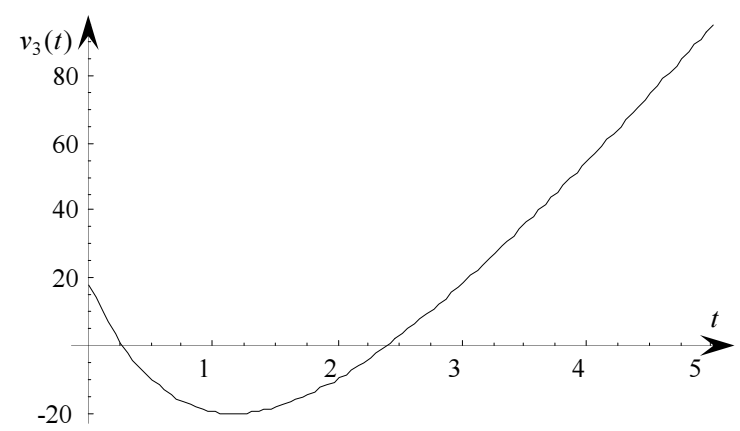

Fig. 3. Graph of the income of system $S_{3}$ of a network

\section{References}

[1] Matalytski M., Kiturko O., Czhornaja N., Finding expected incomes in HM-network with priority requests and linear time-dependent intensity of their service, Scientific Research of the Institute of Mathematics and Computer Science 2011, 2(10), 179-190.

[2] Matalytski M., Tikhonenko O., Koluzaeva E., Queueing systems and networks: analysis and applications, GrSU, Grodno 2011 (in Russian). 